CTP TAMU-13/97

LPTENS-97/07

hep-th/9703012

March 1997

\title{
Towards a Field Theory of F-theory
}

\author{
N. Khviengia ${ }^{\dagger}$, Z. Khviengia ${ }^{\dagger}$, H. Lü ${ }^{\ddagger}$, and C.N. Pope ${ }^{\dagger 1}$ \\ ${ }^{\dagger}$ Center for Theoretical Physics \\ Texas AEM University, College Station, Texas 77843 \\ ${ }^{\ddagger}$ Laboratoire de Physique Théorique de l'École Normale Supérieure ${ }^{2}$ \\ 24 Rue Lhomond - 75231 Paris CEDEX 05
}

\begin{abstract}
$\underline{\text { ABSTRACT }}$
We make a proposal for a bosonic field theory in twelve dimensions that admits the bosonic sector of eleven-dimensional supergravity as a consistent truncation. It can also be consistently truncated to a ten-dimensional Lagrangian that contains all the BPS $p$-brane solitons of the type IIB theory. The mechanism allowing the consistent truncation in the latter case is unusual, in that additional fields with an off-diagonal kinetic term are nonvanishing and yet do not contribute to the dynamics of the ten-dimensional theory. They do, however, influence the oxidation of solutions back to twelve dimensions. We present a discussion of the oxidations of all the basic BPS solitons of M-theory and the type IIB string to $D=12$. In particular, the NS-NS and R-R strings of the type IIB theory arise as the wrappings of membranes in $D=12$ around one or other circle of the compactifying 2 -torus.
\end{abstract}

\footnotetext{
${ }^{1}$ Research supported in part by DOE Grant DE-FG03-95ER40917

${ }^{2}$ Unité Propre du Centre National de la Recherche Scientifique, associée à l'École Normale Supérieure et à l'Université de Paris-Sud
} 


\section{Introduction}

For a variety of reasons, most notable of which is its $S L(2, Z)$ symmetry, it has been proposed that the type IIB string in ten dimensions may have its origin in a twelve-dimensional theory known as F-theory [1, 2]. This proposal is parallel to the suggestion that the type IIA string has its origin in eleven-dimensional M-theory [3]. In this latter case, it is clear that the low-energy limit of M-theory is the long-known eleven-dimensional supergravity [4]. Indeed, one can see evidence for the eleven-dimensional origin of the type IIA string purely at the level of its low-energy effective action, in that this ten-dimensional type IIA supergravity arises as a consistent truncation that retains just the massless sector of the compactification of $D=11$ supergravity on a circle [5, 6].

In the case of the type IIB string and F-theory, we do not have the benefit of already knowing the form of the low-energy effective theory in twelve dimensions. Indeed, we know that there cannot be any ordinary supergravity field theory in $D=12$. Thus we have a less clear-cut starting point for investigations of the compactifications of the hypothetical F-theory. One may hope nevertheless that even at a purely field-theoretic level, there might exist some twelve-dimensional theory that could be compactified to ten dimensions on a 2-torus, in such a way that type IIB supergravity would emerge as a consistent truncation. It is crucial that the truncation should be consistent, in order that solutions of the type IIB supergravity will also be solutions of the equations of motion of the twelve-dimensional theory in which it is embedded. The twelve-dimensional theory should also be capable of being consistently truncated to $D=11$ supergravity.

In this paper we shall study this problem from the field theoretic point of view, along the lines discussed above. In particular, we shall present arguments that seem to lead rather naturally to a candidate for the twelve-dimensional field theory. To be more precise, we focus our attention on the bosonic sector of the field theory. We first show that it can be consistently truncated to the bosonic sector of $D=11$ supergravity, after compactifying from $D=12$ on a circle. We then consider instead the compactification from $D=12$ to $D=10$ on a 2 -torus. We show that this admits a consistent truncation to the set of fields considered in the Lagrangian formulation of the type IIB theory in [7], namely the fields of type IIB supergravity but with no duality restriction on the 5 -form field strength. (In [7], the equations of motion following from the Lagrangian with the non-self-dual 5-form can be reduced to the type IIB equations by imposing self-duality as a consistent truncation.) Our dimensionally reduced theory in ten dimensions, after truncating the other unwanted fields, admits type IIB supergravity as a further consistent truncation at the linearised level. To 
be more specific, all interaction terms except the trilinear interaction between the 5 -form and the two 3-form field strengths are correctly reproduced. Thus the subset of type IIB solutions for which this interaction plays no rôle are also solutions of our ten-dimensional equations of motion. A crucial ingredient in the construction turns out to be a dilatonic scalar field in the twelve-dimensional theory, which plays a vital rôle in the subsequent truncation in ten dimensions.

Given embeddings of $D=11$ supergravity and type IIB supergravity in the twelvedimensional theory, one can consider the various BPS-saturated $p$-brane solutions in these lower-dimensional theories, and examine their reinterpretation as solutions in the higher dimension. We are able to carry out this procedure to oxidise the M-branes of M-theory to $D=12$. Although the incompleteness of the embedding of the type IIB theory precludes a full discussion of oxidations from $D=10$, one may nevertheless argue that the oxidation to $D=12$ of those solutions of type IIB gravity for which the trilinear gauge-field interaction plays no rôle may be sensibly discussed. These include all the basic BPS-saturated $p$-branes of the type IIB theory. In particular, we show how the NS-NS and R-R strings of the type IIB theory arise from wrapping membranes in $D=12$ around one or other circle of the compactifying 2-torus.

\section{The low-energy effective Lagrangian for F-theory}

Several authors have discussed the probable field content of a twelve-dimensional theory that could be capable of yielding type IIB supergravity after compactification to ten dimensions [8, 9, 10]. As will be the case in our subsequent considerations, attention has been restricted to the bosonic fields in the theory. It is clear that in order to obtain a 5-form field strength in $D=10$, the simplest assumption would be to include such a 5 -form $G_{5}$ already in $D=12$. For two reasons, this is not yet sufficient, however. Firstly, we need to get two 3-form field strengths in $D=10$, whilst the toroidal reduction of $G_{5}$ would yield only one. Secondly, we need to obtain the right cubic gauge-field interaction term in $D=11$. This would have to come from a topological cubic term in $D=12$, and there is no way to write such a term if one has only $G_{5}$ and its potential $B_{4}$ available. Both of these deficiencies might be cured if a second field strength $F_{4}=d A_{3}$, of rank 4 , is introduced too. Now we may write down a topological term $B_{4} \wedge d A_{3} \wedge d A_{3}$ in $D=12$ [8], which is capable of yielding cubic terms of the right general structures in eleven and ten dimensions. Thus our proposed bosonic field content in $D=12$ now includes the metric $g_{M N}$, and the 3 -form and 4-form potentials $A_{3}$ 
and $B_{4}$.

It is not hard to see that there is still a further deficiency with the above field content. The reason for this can be seen by looking at the way in which Kaluza-Klein reductions work in general. When a field theory is compactified on a circle from $D+1$ dimensions to $D$ dimensions, the metric in the Einstein frame is reduced according to

$$
d s_{D+1}^{2}=e^{2 \alpha \varphi} d s_{D}^{2}+e^{-2(D-2) \alpha \varphi}(d z+\mathcal{A})^{2}
$$

where $d s_{D}^{2}, \varphi$ and $\mathcal{A}=\mathcal{A}_{M} d x^{M}$ are independent of the compactification coordinate $z$, and the constant $\alpha$ is chosen to be $\alpha=\left((2(D-1)(D-2))^{-1 / 2}\right.$ in order to achieve a conventional normalisation for the lower-dimensional kinetic terms. A potential of rank $(n-1)$ is reduced according to

$$
A_{n-1}(x, z) \longrightarrow A_{n-1}(x)+A_{n-2}(x) \wedge d z
$$

This means that if we start from a Lagrangian in $D+1$ dimensions of the form

$$
\mathcal{L}_{D+1}=e R-\frac{1}{2} e(\partial \phi)^{2}-\frac{1}{2 n !} e e^{\hat{a} \phi} F_{n}^{2},
$$

then the resulting Lagrangian in $D$ dimensions will be [11]

$$
\begin{aligned}
\mathcal{L}_{D}= & e R-\frac{1}{2} e(\partial \phi)^{2}-\frac{1}{2} e(\partial \varphi)^{2}-\frac{1}{4} e e^{-2(D-1) \alpha \varphi} \mathcal{F}_{2}^{2} \\
& -\frac{1}{2 n !} e e^{\hat{a} \phi-2(n-1) \alpha \varphi} F_{n}^{2}-\frac{1}{2(n-1) !} e e^{\hat{a} \phi+2(D-n) \alpha \varphi} F_{n-1}^{2},
\end{aligned}
$$

where $\mathcal{F}_{2}=d \mathcal{A}$. Note that the lower-dimensional field strength $F_{n}$ appearing here will acquire a Chern-Simons type correction, with $F_{n}=d A_{n-1}-d A_{n-2} \wedge \mathcal{A}$ in $D$ dimensions. This can be seen by noting that the exterior derivative of (2), organised in terms of vielbein components, is $d A_{n-1}-d A_{n-2} \wedge \mathcal{A}+d A_{n-2} \wedge(d z+\mathcal{A})$. It is these vielbein components which appear in the lower-dimensional kinetic terms.

The strength of a dilaton coupling of the form $e^{\hat{a} \phi+b \varphi} F_{n}^{2}$ can best be expressed by making a rotation to a new canonically-normalised dilatonic scalar $\phi^{\prime}=\left(\hat{a}^{2}+b^{2}\right)^{-1 / 2}(\hat{a} \phi+b \varphi)$, so that we now have $e^{a \phi^{\prime}} F_{n}^{2}$, where $a^{2}=\hat{a}^{2}+b^{2}$. Although the value of the coupling constant $a$ is changed from the value $\hat{a}$ in the higher dimension, there is a nice way to reparameterise the couplings in a way that is preserved under Kaluza-Klein reduction [11]. Thus we introduce a new constant $\Delta$, related to $a$ in $D$ dimensions by

$$
a^{2}=\Delta-\frac{2(n-1)(D-n-1)}{D-2} .
$$

It is not hard to see that the effective dilaton couplings for all field strengths are such that their values of $\Delta$ remain unchanged under Kaluza-Klein reduction. One can also easily 
verify that the new 2-form field strengths $\mathcal{F}_{2}$ that emerge from the dimensional reduction of the metric have $\Delta=4$. In fact, interestingly enough all dilaton coupling constants for all field strengths in maximal supergravities have $\Delta=4$ [11]. This includes cases such as $D=11$ supergravity, where the absence of the dilaton corresponds to having $a=0$, and this translates, using (5), into $\Delta=4$.

With these preliminaries, we can see why the Kaluza-Klein reduction of a twelvedimensional field theory involving just $g_{M N}, A_{3}$ and $B_{4}$ is not capable of admitting type IIB supergravity as a consistent truncation. The reason is that the type IIB theory has $\Delta=4$ for all its dilaton couplings, but the theory in $D=12$ with no dilaton will, using (5), have $\Delta=\frac{21}{5}$ for the 4 -form field strength, and $\Delta=\frac{24}{5}$ for the 5 -form field strength. Unless some truncation of the field content in the dimensionally-reduced theory is performed, the values of $\Delta$ in the higher dimension will persist in the lower dimension. Another possibility is to introduce a dilaton already in $D=12$, in order to change the couplings to $\Delta=4$ already in twelve dimensions. It turns out that this latter procedure is the one that is needed in order to end up with the correct $\Delta=4$ dilaton couplings in ten dimensions. On the other hand, the required $\Delta=4$ coupling of $D=11$ supergravity can be achieved without the need for the dilaton in $D=12$, by setting an appropriate linear combination of the two 4 -form field strengths in eleven dimensions to zero. This truncation can also be described within the framework needed for the ten-dimensional reduction, by simply setting the $D=12$ dilaton to zero as well. Thus we may embed both the eleven-dimensional and ten-dimensional theories in a single theory in $D=12$, where a dilaton is included in order to have couplings with $\Delta=4$. Accordingly, we take as our starting point the twelve-dimensional Lagrangian density

$$
\mathcal{L}_{12}=e R-\frac{1}{2} e(\partial \psi)^{2}-\frac{1}{48} e^{a \psi} F_{4}^{2}-\frac{1}{240} e e^{b \psi} G_{5}^{2}+\lambda B_{4} \wedge d A_{3} \wedge d A_{3}
$$

where $\psi$ is the dilaton, and $a$ and $b$ are constants. The last term is understood to be dualised to give a 0 -form contribution to the Lagrangian density, i.e. it is equal to $\frac{\lambda}{864} \epsilon^{M_{1} \cdots M_{12}} B_{M_{1} \cdots M_{4}} \partial_{M_{5}} A_{M_{6} \cdots M_{8}} \partial_{M_{9}} A_{M_{10} \cdots M_{12}}$.

Using (5), it is easy to see that if the dilaton couplings to the 4-form and 5 -form field strengths are to correspond to the canonical value $\Delta=4$, we must have

$$
a^{2}=-\frac{1}{5} \quad b^{2}=-\frac{4}{5}
$$

It is interesting that to achieve $\Delta=4$, it seems that theories in $D<11$ need real dilaton couplings, the theory in $D=11$ itself needs zero dilaton coupling, and the theories in $D>11$ 
need imaginary dilaton couplings. We shall see later that the imaginary couplings, far from being undesirable, are exactly what is needed in order to make a consistent truncation to the fields of type IIB supergravity possible.

Let us now follow the standard Kaluza-Klein procedure to reduce the twelve-dimensional theory $(\sqrt{6})$, first to $D=11$ and then to $D=10$. In an obvious notation, where $F_{4}$ reduces to $F_{4}, F_{3}^{(i)}, F_{2}^{(i j)}, \ldots$ after compactification on internal circles labelled by $i$, and $G_{5}$ similarly reduces to $G_{5}, G_{4}^{(i)}, G_{3}^{(i j)}, \ldots$, we see from (3) and (44) that the reduction of (6) to $D=11$ gives

$$
\begin{aligned}
e^{-1} \mathcal{L}_{11}= & R-\frac{1}{2}(\partial \psi)^{2}-\frac{1}{2}\left(\partial \phi_{1}\right)^{2}-\frac{1}{48} e^{-\frac{1}{\sqrt{5}} \phi_{1}+a \psi} F_{4}^{2} \\
& -\frac{1}{12} e^{\frac{7}{3 \sqrt{5}} \phi_{1}+a \psi}\left(F_{3}^{(1)}\right)^{2}-\frac{1}{240} e^{-\frac{4}{3 \sqrt{5}} \phi_{1}+b \psi} G_{5}^{2} \\
& -\frac{1}{48} e^{\frac{2}{\sqrt{5}} \phi_{1}+b \psi}\left(G_{4}^{(1)}\right)^{2}-\frac{1}{4} e^{-\frac{10}{3 \sqrt{5}} \phi_{1}}\left(\mathcal{F}_{2}^{(1)}\right)^{2}+e^{-1} \mathcal{L}_{\mathrm{FFA}} .
\end{aligned}
$$

As mentioned above, there will be Chern-Simons type modifications in the expressions for the field strengths $F_{4}$ and $G_{5}$ in eleven dimensions. Their detailed forms may be found by applying the results given in [12]. We shall discuss the cubic interactions $\mathcal{L}_{\mathrm{FFA}}$ later.

After a reduction on a further circle, we will get a Lagrangian in ten dimensions which now has three dilatonic scalars, namely $\psi, \phi_{1}$ and a further scalar $\phi_{2}$ from the $g_{11,11}$ component of the eleven-dimensional metric. It will be convenient to perform an $S O(2)$ rotation on the $\phi_{1}$ and $\phi_{2}$ dilatons, by defining

$$
\phi=\frac{\sqrt{5}}{3} \phi_{1}-\frac{2}{3} \phi_{2}, \quad \varphi=\frac{2}{3} \phi_{1}+\frac{\sqrt{5}}{3} \phi_{2} .
$$

As we shall see below, it is the $\phi$ field that acquires an interpretation as the dilaton of the type IIB theory. The $\varphi$ field, on the other hand, parameterises the volume of the compactifying 2-torus, which is given by $e^{-\frac{2}{\sqrt{5}} \varphi}$. In terms of these rotated scalars, we find that the dimensional reduction of (6) to $D=10$ gives the Lagrangian

$$
\begin{aligned}
e^{-1} \mathcal{L}_{10}= & R-\frac{1}{2}(\partial \psi)^{2}-\frac{1}{2}(\partial \phi)^{2}-\frac{1}{2}(\partial \varphi)^{2}-\frac{1}{2} e^{-2 \phi}(\partial \chi)^{2}-\frac{1}{12} e^{\frac{3}{\sqrt{5}} \varphi+b \psi}\left(G_{3}^{(12)}\right)^{2} \\
& -\frac{1}{240} e^{-\frac{2}{\sqrt{5}} \varphi+b \psi} G_{5}^{2}-\frac{1}{12} e^{\phi+\frac{1}{\sqrt{5}} \varphi+a \psi}\left(F_{3}^{(1)}\right)^{2}-\frac{1}{12} e^{-\phi+\frac{1}{\sqrt{5}} \varphi+a \psi}\left(F_{3}^{(2)}\right)^{2} \\
& -\frac{1}{48} e^{-\frac{3}{2 \sqrt{5}} \varphi+a \psi} F_{4}^{2}-\frac{1}{48} e^{\phi+\frac{1}{2 \sqrt{5}} \varphi+b \psi}\left(G_{4}^{(1)}\right)^{2}-\frac{1}{48} e^{-\phi+\frac{1}{2 \sqrt{5}} \varphi+b \psi}\left(G_{4}^{(2)}\right)^{2} \\
& -\frac{1}{4} e^{\frac{7}{2 \sqrt{5}} \varphi+a \psi}\left(F_{2}^{(12)}\right)^{2}-\frac{1}{4} e^{-\phi-\frac{\sqrt{5}}{2} \varphi}\left(\mathcal{F}_{2}^{(1)}\right)^{2}-\frac{1}{4} e^{\phi-\frac{\sqrt{5}}{2} \varphi}\left(\mathcal{F}_{2}^{(2)}\right)^{2}+e^{-1} \mathcal{L}_{\mathrm{FFA}},
\end{aligned}
$$

where $\chi=\mathcal{A}_{0}^{(12)}$ is the 0 -form potential coming from the dimensional reduction of the Kaluza-Klein vector $\mathcal{A}_{1}^{(1)}$ in $D=11$. There will be Chern-Simons modifications in several of the field strengths appearing here; again, the full details may be found using the general results in [12]. 
Having obtained the complete dimensionally-reduced Lagrangians in $D=11$ and $D=10$ dimensions, we shall now examine the possibility of consistently truncating them to give $D=11$ supergravity and type IIB supergravity respectively. First, we shall consider the truncation to $D=11$ supergravity. This can be performed exactly (at least in the bosonic sector, which we are considering in this paper). In this truncation, the dilatonic scalar $\psi$ which we introduced in twelve dimensions is in fact set to zero. In the truncation of the ten-dimensional Lagrangian (10), on the other hand, it will turn out that the dilaton $\psi$ plays a crucial rôle.

\subsection{Reduction to $D=11$ supergravity}

In this section we shall show that the dimensional reduction of the twelve-dimensional theory to $D=11$ may be truncated to give $D=11$ supergravity. Our starting-point is the eleven-dimensional Lagrangian (8). Obviously, since (8) contains more fields than are present in $D=11$ supergravity, some of them must be set to zero. The important point is that this truncation must be consistent, i.e. setting the fields to zero must be consistent with their equations of motion. We first observe that we may consistently set

$$
G_{5}=F_{3}^{(1)}=\mathcal{F}_{2}^{(1)}=0 .
$$

In general, owing to the Chern-Simons modifications and $\mathcal{L}_{\mathrm{FFA}}$ term, setting a field strength to zero can be inconsistent with the equations of motion. However in this case, setting all three of the field strengths in (11) to zero simultaneously is nevertheless consistent. This follows from the fact that their equations of motion have the general form

$$
\begin{aligned}
\nabla \cdot G_{5} & \sim \epsilon F_{4} F_{3}^{(1)} \\
\nabla \cdot F_{3}^{(1)} & \sim \epsilon G_{5} F_{4}+F_{4} \cdot \mathcal{F}_{2}^{(1)}, \\
\nabla \cdot \mathcal{F}_{2}^{(1)} & \sim G_{5} \cdot G_{4}^{(1)}+F_{4} \cdot F_{3}^{(1)}
\end{aligned}
$$

and thus after imposing (11), the source terms on the right-hand sides of the equations of motion (12) vanish.

Now recall that we are choosing the dilaton coupling constants $a$ and $b$ to satisfy (7), which we solve by taking

$$
a=\frac{i}{\sqrt{5}} \quad b=-\frac{2 i}{\sqrt{5}} .
$$

Let us define the complex field $w=\left(-\phi_{1}+i \psi\right) / \sqrt{2}, \bar{w}=\left(-\phi_{1}-i \psi\right) / \sqrt{2}$, in terms of which the consistently truncated Lagrangian takes the form

$$
e^{-1} \mathcal{L}_{11}=R-\partial w \cdot \partial \bar{w}-\frac{1}{48} e^{\sqrt{\frac{2}{5}} w} F_{4}^{2}-\frac{1}{48} e^{-2 \sqrt{\frac{2}{5}} w}\left(G_{4}^{(1)}\right)^{2}+\lambda e^{-1} B_{3}^{(1)} \wedge d A_{3} \wedge d A_{3} .
$$


Note that the anti-holomorphic part $\bar{w}$ of the complex scalar field appears only in the off-diagonal kinetic term, and thus the equations of motion for $w$ and $\bar{w}$ are

$$
\begin{aligned}
\nabla^{2} w & =0 \\
\nabla^{2} \bar{w} & =\frac{1}{48} \sqrt{\frac{2}{5}}\left(e^{\sqrt{\frac{2}{5}} w} F_{4}^{2}-2 e^{-2 \sqrt{\frac{2}{5}} w}\left(G_{4}^{(1)}\right)^{2}\right) .
\end{aligned}
$$

It should be emphasised that $w$ and $\bar{w}$ are to be treated as independent fields. Clearly, we may consistently set $w=0$, and while (15) implies that $\bar{w}$ will in general be non-zero, it is a kind of "phantom" field that does not influence any of the other eleven-dimensional equations of motion.

From (14) the equations of motion for $F_{4}$ and $G_{4}^{(1)}$, having set $w=0$, become

$$
\begin{aligned}
d * F_{4} & =2 \lambda G_{4}^{(1)} \wedge F_{4} \\
d * G_{4}^{(1)} & =\lambda F_{4} \wedge F_{4} .
\end{aligned}
$$

In order to obtain $D=11$ supergravity, it is necessary to reduce the remaining system of fields further, so that in particular we have only a single independent 4-form field strength, rather than two. We may do this by taking $F_{4}$ and $G_{4}^{(1)}$ to be proportional, again making sure that this truncation of the theory is consistent with the equations of motion. Thus we may define

$$
F_{4}=\widetilde{F}_{4} \sin \beta \quad G_{4}=\widetilde{F}_{4} \cos \beta,
$$

where the constant angle $\beta$ is determined by the consistency of the two equations (16), which then imply $F_{4}=\sqrt{2} G_{4}^{(1)}$ and hence

$$
\cos \beta=\frac{1}{\sqrt{3}}, \quad \sin \beta=\sqrt{\frac{2}{3}} .
$$

Substituting into (15), we find that the right-hand side of the equation of motion for $\bar{w}$ in fact now vanishes, and thus we may solve $\nabla^{2} \bar{w}=0$ by taking $\bar{w}=0$. By this means we

\footnotetext{
${ }^{1}$ Another way of describing the theory is by starting out with a dilatonic scalar in $D=12$ with the "wrong sign" for its kinetic term. Effectively we may define $\theta=i \psi$, so that in $D=12$ we have

$$
\mathcal{L}_{12}=e R+\frac{1}{2} e(\partial \theta)^{2}-\frac{1}{48} e e^{\frac{1}{\sqrt{5}} \theta} F_{4}^{2}-\frac{1}{240} e e^{-\frac{2}{\sqrt{5}} \theta} G_{5}^{2}+\lambda B_{4} \wedge d A_{3} \wedge d A_{3} .
$$

Now, the combinations of $\phi_{1}$ and $\theta$ in $D=11$ will be of the form $w=\left(-\phi_{1}+\theta\right) / \sqrt{2}$ and $\tilde{w}=\left(-\phi_{1}-\theta\right) / \sqrt{2}$, again with the desired off-diagonal kinetic term $-\partial w \cdot \partial \tilde{w}$ in the eleven-dimensional Lagrangian. In this formulation it is clear that $w$ and $\tilde{w}$ are independent, and that we may set $w=0$ while $\tilde{w}$ is non-zero. Since the complex description is formally equivalent to this one, we shall for simplicity work with the $w$ and $\bar{w}$ variables.
} 
arrive at a consistent truncation of the dimensionally reduced eleven-dimensional theory described by (8), which is described by the Lagrangian

$$
\mathcal{L}=e R-\frac{1}{48} e \widetilde{F}_{4}^{2}+\frac{2}{3 \sqrt{3}} \lambda \widetilde{A}_{3} \wedge d \widetilde{A}_{3} \wedge d \widetilde{A}_{3}
$$

where $\widetilde{F}_{4}=d \widetilde{A}_{3}$. Comparison with the Lagrangian for the bosonic sector of $D=11$ supergravity shows that we must take the coefficient $\lambda$, introduced in (6), to be

$$
\lambda=\frac{1}{4} \sqrt{3}
$$

Thus we have succeeded in embedding the bosonic sector of $D=11$ supergravity as a consistent truncation in the twelve-dimensional theory described by (6).

It is worth emphasising that the dilaton $\psi$ in twelve dimensions played an essential rôle in ensuring the consistency of the truncation of the fields given in (11), since the complex field $\bar{w}=\left(-\phi_{1}-i \psi_{1}\right) / \sqrt{2}$ had to be non-zero in order to satisfy its equation of motion (15). The fact that the further truncation (17) eventually led to the simpler situation where $\psi$ vanished was a consequence of the apparent coincidence that the ratio between $F_{4}$ and $G_{4}^{(1)}$ that was required for consistency of the equations of motion for these two fields happened to be the same as the ratio for which the right-hand side of the equation for $\bar{w}$ in (15) vanished. The situation is different, however, in the truncation of the fields in the ten-dimensional theory (10) to those of type IIB supergravity. In the next section, we show that in this case the consistency of the truncation to the desired set of fields requires that $\psi$ be non-zero.

\subsection{Reduction to type IIB supergravity}

In this section we shall attempt the truncation of the ten-dimensional Lagrangian (10) to give type IIB supergravity. We begin by setting to zero those field strengths that are not present in type IIB supergravity, namely

$$
F_{4}=G_{4}^{(1)}=G_{4}^{(2)}=G_{3}^{(12)}=\mathcal{F}_{2}^{(1)}=\mathcal{F}_{2}^{(2)}=F_{2}^{(12)}=0
$$

As in the eleven-dimensional case, we find from a careful investigation of the equations of motion for these fields that their non-linear source terms, arising because of the ChernSimon modifications and $\mathcal{L}_{\mathrm{FFA}}$ term, vanish when the conditions (21) are imposed, and thus the truncation of these fields is a consistent one. The Lagrangian (10) is now reduced to

$$
\begin{aligned}
e^{-1} \mathcal{L}_{10}= & R-\frac{1}{2}(\partial \psi)^{2}-\frac{1}{2}(\partial \phi)^{2}-\frac{1}{2}(\partial \varphi)^{2}-\frac{1}{2} e^{-2 \phi}(\partial \chi)^{2}-\frac{1}{240} e^{-\frac{2}{\sqrt{5}} \varphi+b \psi} G_{5}^{2} \\
& -\frac{1}{12} e^{\phi+\frac{1}{\sqrt{5}} \varphi+a \psi}\left(F_{3}^{(1)}\right)^{2}-\frac{1}{12} e^{-\phi+\frac{1}{\sqrt{5}} \varphi+a \psi}\left(F_{3}^{(2)}\right)^{2}+e^{-1} \mathcal{L}_{\mathrm{FFA}} .
\end{aligned}
$$


After taking into account the Chern-Simons modifications to the various field strengths, as discussed above, we find that $F_{3}^{(1)}$ and $F_{3}^{(2)}$ are given by

$$
F_{3}^{(1)}=d A_{2}^{(1)}, \quad F_{3}^{(2)}=d A_{2}^{(2)}-\chi d A_{2}^{(1)} \text {. }
$$

These are precisely the structures of the NS-NS and R-R 3-forms respectively, in type IIB supergravity. Note that before the truncations (21) there are in total three 3-form field strengths, namely $G_{3}^{(12)}, F_{3}^{(1}$ and $F_{3}^{(2)}$. Since $G_{3}^{(12)}$ is a singlet under the $S L(2, R)$ symmetry, it is clear that it should be truncated from the ten-dimensional theory; the remaining two 3 -forms form the required doublet under $S L(2, R)$.

We would like to be able to truncate the scalars $\varphi$ and $\psi$ also, since only the dilaton $\phi$ should remain in the type IIB theory. However, it is clear from (22) that setting $\varphi$ and $\psi$ to zero would be inconsistent with their equations of motion, since the remaining field strengths act as sources for these scalars. At this point, we recall that the constants $a$ and $b$ should satisfy the conditions given in (7). In particular, we shall take them to be given by (13). If we then define the complex field $u$, and its conjugate $\bar{u}$, by

$$
u=\frac{1}{\sqrt{2}}(\varphi+i \psi), \quad \bar{u}=\frac{1}{\sqrt{2}}(\varphi-i \psi),
$$

then the truncated Lagrangian (22) takes the form

$$
\begin{aligned}
e^{-1} \mathcal{L}_{10}= & R-\partial u \cdot \partial \bar{u}-\frac{1}{2}(\partial \phi)^{2}-\frac{1}{2} e^{-2 \phi}(\partial \chi)^{2}-\frac{1}{240} e^{-2 \sqrt{\frac{2}{5}} u} G_{5}^{2} \\
& -\frac{1}{12} e^{\sqrt{\frac{2}{5}} u}\left(e^{\phi}\left(F_{3}^{(1)}\right)^{2}+e^{-\phi}\left(F_{3}^{(2)}\right)^{2}\right)+e^{-1} \mathcal{L}_{\mathrm{FFA}} .
\end{aligned}
$$

The equations of motion for $u$ and $\bar{u}$ are

$$
\begin{aligned}
\nabla^{2} u & =0 \\
\nabla^{2} \bar{u} & =\frac{1}{12} \sqrt{\frac{2}{5}} e^{\sqrt{\frac{2}{5}} u}\left(e^{\phi}\left(F_{3}^{(1)}\right)^{2}+e^{-\phi}\left(F_{3}^{(2)}\right)^{2}\right)-\frac{1}{120} \sqrt{\frac{2}{5}} e^{-2 \sqrt{\frac{2}{5}} u} G_{5}^{2} .
\end{aligned}
$$

Note that $u$ and $\bar{u}$ are treated as independent variables in these equations (just like the $w$ and $\bar{w}$ fields in $D=11)$, and they are both $S L(2, R)$ invariant. We see that it is consistent to set $u=0$, while $\bar{u}$ will in general be non-zero. However, the important point is that $\bar{u}$ appears in the Lagrangian only in the off-diagonal kinetic term $\partial u \cdot \partial \bar{u}$, and so $\bar{u}$ is a phantom field which, even though non-zero, has no influence on the solutions for the other fields in the ten-dimensional theory.

In terms of $\varphi$ and $\psi$, we see from (24) that the volume $e^{-\frac{2}{\sqrt{5}} \varphi}$ of the compactifying 2 -torus is not constant in general. However, it does not contribute to the dynamics of the ten-dimensional theory, and it does not interfere with the $S L(2, R)$ symmetry. It should 
again be emphasised that although it might a priori have seemed more natural to demand that $\varphi$ simply be non-dynamical, it is not possible to do this because its equation of motion requires that it be non-constant in general. Of course if one considers only the scalar sector of the type IIB theory, where the higher-degree field strengths are zero, then the inconsistency of setting $\varphi=$ const. in the full theory is not apparent. Thus the necessity of introducing the dilaton $\psi$ in $D=12$, which resolves this inconsistency, is not seen if one restricts attention to the scalar sector. For this reason the discussion in [9], where the 3 -form and 5-form field strengths were taken to be zero, does not encounter inconsistencies. However the theory in $D=12$ with the field content $\left\{g_{M N}, A_{3}, B_{4}\right\}$ proposed in [8, 9, 10] will run into this inconsistency problem. Note that the inconsistency would arise not only in the $T^{2}$ compactification of the twelve-dimensional theory, but also in all the compactifications on elliptically-fibred manifolds. It should be emphasised also that despite the fact that $u$ and $\bar{u}$ are treated as independent variables, the volume parameter $\varphi$ will always be real, and it is only the dilaton $\psi$ that is imaginary. As we remarked earlier, this also could be made real by replacing $\psi$ by a field $\theta=i \psi$.

In order to obtain the precise form of the ten-dimensional type IIB supergravity theory, we should like to be able to perform a further truncation, by setting the anti-self-dual part of the 5 -form field strength $G_{5}$ to zero. In fact the Lagrangian (25), after taking care of the truncation of $u$ and the decoupling of $\bar{u}$ discussed above, is superficially of the form of the Lagrangian obtained in [7], which, when properly used by imposing self-duality of $G_{5}$ after obtaining the equations of motion, describes type IIB supergravity. However, there is unfortunately a discrepancy, namely that our field strength $G_{5}$ in $D=10$ is given simply by $G_{5}=d B_{4}$, whereas the field strength in [0] has a Chern-Simons correction, with $G_{5}=d B_{4}+\lambda \epsilon_{i j} A_{2}^{(i)} \wedge d A_{2}^{(j)}$. This means that since our equation of motion and Bianchi identity for $G_{5}$ are of the form

$$
d * G_{5}=\lambda \epsilon_{i j} d A_{2}^{(i)} \wedge d A_{2}^{(j)}, \quad d G_{5}=0,
$$

we cannot in general, unlike in [7] where $d G_{5}=\lambda \epsilon_{i j} d A_{2}^{(i)} \wedge d A_{2}^{(j)}$, consistently impose the self-duality condition $G_{5}=* G_{5}$.

It should be remarked that we would not necessarily have to be able to set the anti-selfdual 5-form to zero, provided that it decoupled from the other equations of motion. Let us define $H_{5}^{( \pm)}=G_{5} \pm * G_{5}$, where $H_{5}^{(+)}$is self-dual and $H_{5}^{(-)}$is anti-self-dual. Then the self-dual 5-form $H_{5}^{(+)}$satisfies precisely the same equation of motion and Bianchi identity 
as in type IIB supergravity, namely 15

$$
d * H_{5}^{(+)}=d H_{5}^{(+)}=\lambda \epsilon_{i j} d A_{2}^{(i)} \wedge d A_{2}^{(j)} .
$$

Unfortunately, the anti-self-dual 5-form $H_{5}^{(-)}$does not decouple from the full equations of motion of type IIB supergravity; it appears in the equations of motion for the 3 -form field strengths, namely

$$
d * F_{3}^{(i)}=2 \lambda G_{5} \wedge F_{3}^{(j)} \epsilon_{i j}=\lambda\left(H_{5}^{(+)}+H_{5}^{(-)}\right) \wedge F_{3}^{(j)} \epsilon_{i j}
$$

The truncation of the twelve-dimensional Lagrangian to the type IIB theory in $\mathrm{D}=10$ is therefore consistent only up to linear order when the 5-form field strength is involved. However, the problem is avoided altogether in the case of configurations for which

$$
\epsilon_{i j} d A_{2}^{(i)} \wedge d A_{2}^{(j)}=0
$$

For precisely the same reason, it is possible to consider a "truncated" Lagrangian for type IIB supergravity without the 5-form field strength [16]. In general such a truncation is inconsistent, owing to the equations of motion and Bianchi identity for the self-dual 5-form field strength $H_{5}^{(+)}$, given in (29); however, it becomes consistent if (31) is satisfied.

This condition can easily be seen to be satisfied by all singly-charged BPS-saturated p-brane solitons, and their $S L(2, R)$ duality multiplets, in the type IIB theory, and so such solutions of the type IIB theory (which preserve half the supersymmetry) are also solutions of the dimensionally-reduced Lagrangian (25) with $u=0$. In type IIB supergravity, there is, first of all, a self-dual 3-brane, which makes use of the R-R self-dual 5-form field strength. The solution is a singlet under the $S L(2, R)$ symmetry, and all the other field strengths and the dilaton $\phi$ are zero. Thus we may consistently impose the self-dual constraint in our dimensionally-reduced Lagrangian in this case. For the remaining single-charge $p$-branes and their $S L(2, R)$ multiplets, using 3-form or 1-form field strengths, the constraint (31) is again satisfied and the 5-form field strength is zero. It follows that the corresponding truncated equations of motion are precisely the same as those of type IIB supergravity. Thus all the BPS-saturated extremal $p$-branes of the type IIB theory are contained in the dimensionally-reduced Lagrangian coming from $D=12$. Further analysis shows that in fact all BPS solutions in all dimensional reductions of the type IIB theory also satisfy (31) in $D=10$, and thus they are also all solutions of our ten-dimensional theory. These include multiply-charged solutions that preserve smaller fractions of the supersymmetry, as well as the singly-charged ones that preserve half the supersymmetry. 
Finally, we remark that we could also take the full ten-dimensional theory (10), and perform an alternative consistent truncation that would give the bosonic sector of type IIA supergravity. Of course this would be nothing but a restatement in $D=10$ of the truncations that we performed in $D=11$ to get eleven-dimensional supergravity.

\section{Oxidation of M-branes to $D=12$}

In this section, we consider the BPS-saturated membrane and 5-brane solutions of the lowenergy effective limit of M-theory, and examine their oxidations to the twelve-dimensional field theory presented in the previous section. It is, of course, crucial that we have been able to embed $D=11$ supergravity as a consistent truncation of the theory in $D=12$, in order that this notion of oxidation should have a well-defined meaning.

We begin with the electrically-charged membrane in $D=11$, which takes the form 13

$$
\begin{aligned}
d s_{11}^{2} & =H^{-2 / 3}\left(-d t^{2}+d x^{i} d x^{i}\right)+H^{1 / 3} d y^{m} d y^{m} \\
F_{m \mu \nu \rho} & =\epsilon_{\mu \nu \rho} \partial_{m} H^{-1}
\end{aligned}
$$

where $i=1,2$ and $H$ is harmonic in the 8-dimensional transverse space of the $y^{m}$ coordinates. The simplest single-membrane solution has $H=1+k r^{-6}$, where $r^{2}=y^{m} y^{m}$. As we saw in the previous section, the consistent truncation of (8) to $D=11$ supergravity implies that $w=\bar{w}=0$, and hence $\psi=\phi_{1}=0$. Using (1), we therefore find that the oxidation of the eleven-dimensional membrane gives simply

$$
d s_{12}^{2}=H^{-2 / 3}\left(-d t^{2}+d x^{i} d x^{i}\right)+H^{1 / 3} d y^{m} d y^{m}+d z_{1}^{2}
$$

Since the additional term $d z_{1}^{2}$ does not have the harmonic functional dependence either of the membrane world-volume or of the transverse space, it describes neither a 3-brane nor a line of membranes in $D=12$.

If we instead begin from the 5-brane solution in M-theory, we have [14]

$$
\begin{aligned}
d s_{11}^{2} & =H^{-1 / 3}\left(-d t^{2}+d x^{i} d x^{i}\right)+H^{2 / 3} d y^{m} d y^{m} \\
F_{m n p q} & =\epsilon_{\text {mnpqr }} \partial_{r} H
\end{aligned}
$$

where $i=1, \ldots, 5$ and $H$ is harmonic in the 5 -dimensional transverse space. The oxidation to $D=12$ is simply given by

$$
d s_{12}^{2}=H^{-1 / 3}\left(-d t^{2}+d x^{i} d x^{i}\right)+H^{2 / 3} d y^{m} d y^{m}+d z_{1}^{2}
$$


Again, this describes neither a 6-brane nor a line of 5-branes in $D=12$.

Note that the M-branes supported by the field strength $\widetilde{F}_{4}$ in fact carry charges associated both with $F_{4}$ and $G_{4}^{(1)}$. However, owing to the absence of a dilaton in $D=11$, the M-branes cannot be viewed as bound states of these two charges with zero binding energy, unlike the case of the self-dual 3-brane in $D=10$ which we shall discuss in section 4 . Thus the twelve-dimensional interpretation of M-branes is somewhat obscured. The membrane in $D=11$, when wrapped around the 11'th coordinate, gives rise to the NS-NS string in type IIA theory, and has a quite different interpretation in $D=12$ from the NS-NS string in type IIB, which, as we shall discuss in section 4 , arises as a line of membranes in $D=12$, wrapped around the $12^{\prime}$ th coordinate $z_{1}$. This may not be surprising since after all the NS-NS string in type IIA is intrinsically different from the NS-NS string in type IIB in $D=10$. They do, however, become equivalent owing to T-duality upon compactification to $D=9$.

\section{Oxidation of type IIB $p$-branes to $D=12$}

To begin, we shall consider the string solution in $D=10$, supported by the NS-NS 3 -form field strength $F_{3}^{(1)}$. The equations of motion for the metric, $\phi$ and $F_{3}^{(1)}$ are the standard ones for the type IIB string, giving the usual string solution

$$
\begin{aligned}
d s_{10}^{2} & =H^{-3 / 4}\left(-d t^{2}+d x^{2}\right)+H^{1 / 4} d y^{m} d y^{m} \\
e^{\phi} & =H^{1 / 2}, \quad F_{m \mu \nu}=\epsilon_{\mu \nu} \partial_{m} H^{-1},
\end{aligned}
$$

where $H$ is an harmonic function in the 8-dimensional transverse space described by the $y^{m}$ coordinates. For an isotropic string, the harmonic function is given by $H=1+k r^{-6}$. In order to oxidise the solution (36) back to $D=12$, we need to obtain the solution for $\bar{u}$. The equation of motion (27) for $\bar{u}$ becomes

$$
\nabla^{2} \bar{u}=\frac{1}{12} \sqrt{\frac{2}{5}} e^{\phi}\left(F_{3}^{(1)}\right)^{2}
$$

which, after substituting the form of the solution (36), is readily seen to imply that $\bar{u}=$ $\frac{1}{\sqrt{10}} \log H$. Since the truncation to the type IIB theory sets $u=0$, it follows from (24) that we have $e^{2 \sqrt{5} \varphi}=H$. Thus from (9) and (36), we find that the Kaluza-Klein scalars $\phi_{1}$ and $\phi_{2}$ coming from the reduction on the 2 -torus are given by

$$
e^{\phi_{1}}=H^{\frac{7}{6 \sqrt{5}}}, \quad e^{\phi_{2}}=H^{-1 / 6} .
$$


Finally, tracing back through the steps of dimensional reduction of the metric using (1), we arrive at the twelve-dimensional oxidation of the type IIB NS-NS string metric

$$
d s_{12}^{2}=H^{-7 / 10}\left(-d t^{2}+d x^{2}+d z_{1}^{2}\right)+H^{3 / 10}\left(d y^{m} d y^{m}+d z_{2}^{2}\right) .
$$

Here, for simplicity, we have taken the two additional dimensions to be spacelike. At least in some versions of F-theory, it would be more natural to perform a Wick rotation on one of them, to give a second timelike coordinate (similar remarks apply to the additional coordinate $z_{1}$ in the oxidations of M-theory discussed in the previous section). Note that the metric (39) describes a twelve-dimensional membrane (i.e. an F-brane) with world volume coordinates $\left(t, x, z_{1}\right)$, and with charges uniformly distributed along the transverse-space coordinate $z_{2}$. This result is understandable, since the NS-NS 3-form field strength $F_{3}^{(1)}$ comes from the dimensional reduction of the 4 -form field strength $F_{4}$ in $D=12$, which admits a membrane solution. To get the string in $D=10$ from a membrane in $D=12$, one step of vertical dimensional reduction and one step of diagonal dimensional reduction are necessary.

Now let us see how the ten-dimensional R-R string solution oxidises to $D=12$. In this case, the solution is supported by the R-R 3-form field strength $F_{3}^{(2)}$. The metric and the field strength in the solution in $D=10$ are the same as those given in (36), but the dilaton is given by $e^{-\phi}=H^{1 / 2}$. Since now the equation of motion for $\bar{u}$ is $\nabla^{2} \bar{u}=\frac{1}{12} \sqrt{\frac{2}{5}} e^{-\phi}\left(F_{3}^{(2)}\right)^{2}$, it follows that the solution for $\varphi$ is identical to the NS-NS case, namely $e^{2 \sqrt{5} \varphi}=H$. This is not surprising since as we observed in section 2 , both $u$ and $\bar{u}$ are $S L(2, R)$ invariant. From these expressions for $\phi$ and $\varphi$, one can easily convert to the $\left(\phi_{1}, \phi_{2}\right)$ basis using (9). Thus the twelve-dimensional metric for the R-R string solution becomes

$$
d s_{12}^{2}=H^{-7 / 10}\left(-d t^{2}+d x^{2}+d z_{2}^{2}\right)+H^{3 / 10}\left(d y^{m} d y^{m}+d z_{1}^{2}\right) .
$$

This describes a line of membranes uniformly distributed along the $z_{1}$ direction. It is interesting to compare the two twelve-dimensional metrics (39) and (40). When the twelvedimensional membrane wraps around the 12 'th coordinate $z_{1}$, it gives rise to an NS-NS string in the ten-dimensional type IIB theory, whereas when the membrane wraps instead around the 11 'th coordinate $z_{2}$, it gives rise to an R-R string. This is consistent with the proposition that the non-perturbative $S L(2, Z)$ symmetry [16] of the type IIB theory, which rotates between the NS-NS and R-R strings, is the symmetry of the 2-torus on which the F-theory is compactified.

We have seen that the NS-NS and R-R strings of the type IIB theory arise as twelvedimensional membranes wrapped around different circles in the 2-torus. Thus the $S L(2, Z)$ 
symmetry of the 2 -torus is reflected naturally in the $S L(2, Z)$ symmetry that relates the NSNS and R-R strings. The membrane in $D=12$ is supported by the electric charge carried by the 4 -form field strength $F_{4}$. The two string solutions, corresponding to the wrapping of the membrane around either $z_{1}$ or $z_{2}$, are understood from the ten-dimensional point of view as carrying charge under either $F_{3}^{(1)}$ or $F_{3}^{(2)}$. There is also a 3-brane solution in $D=12$, with electric charge carried by the 5 -form $G_{5}$. (This will have a $(2,2)$ world-volume signature if the signature of the spacetime is $(10,2)$.) This 3-brane can be wrapped around the entire 2-torus to also give a string in $D=10$. However in this case it is a singlet under $(S L(2, R)$, and hence is not part of the type IIB spectrum. Indeed, it corresponds to a string supported by the singlet 3 -form field strength $G_{3}^{(12)}$ that we truncated from the dimensionally-reduced theory in $D=10$ in order to retain only the fields of the type IIB theory. [? Thus the $S L(2, R)$ structure of the type IIB strings seems to be explained naturally in terms of a membrane origin in $D=12$, rather than the 3 -brane origin discussed in [2]. The situation is precisely analogous to that of the $S L(2, R)$ doublet of string solitons in maximal nine-dimensional supergravity; one carries an NS-NS charge, whilst the other carries an R-R charge. They are obtained from M-theory by wrapping the membrane around one or other of the circles on the compactifying 2-torus.

It is interesting to look at the more general family of $(p, q)$ string solutions in the type IIB theory [16], where the NS-NS and R-R 3-forms carry charges $p$ and $q$ respectively. These solutions can be obtained by performing an $S L(2, R)$ rotation of the pure $(1,0)$ NSNS string. The ten-dimensional metric is invariant under this transformation, and is the same as the one given in (36). The dilaton $\phi$ and axion $\chi$ are now given by

$$
e^{-\phi}=a^{2} H^{-1 / 2}+b^{2} H^{1 / 2}, \quad \chi=\frac{a c+b d H}{a^{2}+b^{2} H},
$$

where $a d-b c=1$. The solution for $\bar{u}$, being $S L(2, R)$ invariant, is unchanged by this transformation. After oxidising back to $D=12$, we obtain the metric

$$
\begin{aligned}
d s_{12}^{2}= & H^{-7 / 10}\left(-d t^{2}+d x^{2}\right)+H^{3 / 10} d y^{m} d y^{m}+\left(a^{2}+b^{2} H\right)^{-1} H^{3 / 10} d z_{2}^{2} \\
& +\left(a^{2}+b^{2} H\right) H^{-7 / 10}\left(d z_{1}+\frac{a c+b d H}{a^{2}+b^{2} H} d z_{2}\right)^{2} .
\end{aligned}
$$

\footnotetext{
${ }^{2}$ Note that this wrapping of the 3-brane in $D=12$ around the entire 2-torus does not correspond to the type IIA string either. As we saw in the previous sections, the 4-form field strength of $D=11$ supergravity is a linear combination of $G_{4}^{(1)}$ and $F_{4}$, and thus neither the membrane in $D=11$ nor its diagonal reduction to the string in type IIA has its origin in any simple F-brane solution in $D=12$. Instead, the solution is given by (33).
} 
An analogous analysis applies to the NS-NS and R-R 5-branes. They are given by

$$
d s_{10}^{2}=H^{-1 / 4}\left(-d t^{2}+d x^{i} d x^{i}\right)+H^{3 / 4} d y^{m} d y^{m}, \quad F_{m n p}=\epsilon_{m n p r} \partial_{r} H
$$

together with $e^{-\phi}=H^{1 / 2}$ for the NS-NS 5-brane and $e^{\phi}=H^{1 / 2}$ for the R-R 5-brane. Here $H$ is an harmonic function on the four-dimensional transverse space of the coordinates $y^{m}$. It is straightforward, using (26), (27) and (24), to show that $e^{-2 \sqrt{5} \varphi}=H$. Tracing back through the steps of dimensional reduction, we find that the metrics for the NS-NS and R-R 5 -branes in $D=12$ are given by

$$
\begin{aligned}
& d s_{12}^{2}=H^{-3 / 10}\left(-d t^{2}+d x^{i} d x^{i}+d z_{1}^{2}\right)+H^{7 / 10}\left(d y^{m} d y^{m}+d z_{2}^{2}\right), \\
& d s_{12}^{2}=H^{-3 / 10}\left(-d t^{2}+d x^{i} d x^{i}+d z_{2}^{2}\right)+H^{7 / 10}\left(d y^{m} d y^{m}+d z_{1}^{2}\right),
\end{aligned}
$$

respectively. As one would expect from our previous discussion for strings, both the NS-NS and R-R 5-branes in $D=12$ are dimensional reductions of a line of 6 -branes in $D=12$, supported by a magnetic charge for the 4 -form field strength $F_{4}$. When the 6-brane wraps around $z_{1}$, it gives rise to the NS-NS 5-brane in the type IIB theory; when it wraps instead around $z_{2}$, the resulting solution is the R-R 5-brane.

There are three more $p$-branes in the $D=10$ type IIB theory, namely the self-dual 3-brane using the self-dual 5-form field strength [17, 18], and the instanton and 7-brane using the 1-form field strength $\partial \chi[19]$. For all of these solutions, it follows from (26) and (27) that we have $u=0=\bar{u}$. Let us first consider the the self-dual 3-brane, in which case all the dilatons $\left(\phi_{1}, \phi_{2}, \psi\right)$ are zero, and so the twelve-dimensional metric is given by

$$
d s_{12}^{2}=d s_{10}^{2}+d z_{1}^{2}+d z_{2}^{2},
$$

where $d z_{10}^{2}$ is the ten-dimensional metric of the self-dual 3-brane. This solution is obviously consistent with the fact that the self-dual 3-brane is invariant under the non-perturbative $S L(2, Z)$ symmetry of the type IIB string. Naively, one might expect that the self-dual 3-brane could be viewed as a bound state of electric and magnetic 3-branes, which could be oxidised to a 3 -brane and a 5 -brane in $D=12$ respectively, and hence that the self-dual 3 -brane could be viewed as an intersection of a 3 -brane and a 5 -brane in $D=12$. However, as was observed in [20], the self-dual 3-brane in type IIB theory has $\Delta=4$, and is therefore a basic state itself which cannot be viewed as a bound state of the $\Delta=4$ electric and magnetic 3-branes with zero binding energy. In fact, it was shown in [12] that the metric of a dyonic 3-brane in a non-self-dual theory in $D=10$ is given by

$$
d s_{10}^{2}=\left(1+k r^{-6}\right)^{-1 / 2}\left(-d t^{2}+d x^{i} d x^{i}\right)+\left(1+k r^{-6}\right)^{1 / 2}\left(d r^{2}+r^{2} d \Omega_{5}\right),
$$


where $k=\sqrt{Q_{e}^{2}+Q_{m}^{2}}$, with $Q_{e}$ and $Q_{m}$ the electric and magnetic charges. Thus the selfdual 3-brane can instead be viewed as a bound state with positive binding energy, which cannot be interpreted as an intersection of $p$-branes in higher dimensions [21].

Finally, we have the instanton solution, which oxidises to a pp-wave in $D=12$ [9], and the 7-brane solution. In this latter case, if a 24-centre configuration is chosen, the $D=12$ metric contains a K3 metric, which can be viewed as a 2-torus bundle over a 2-sphere [2].

\section{Discussion and Conclusions}

In this paper, we have shown that there exists a possible candidate field theory for F-theory in $D=12$, whose dimensional reduction to $D=11$ admits a consistent truncation to the bosonic sector of eleven-dimensional supergravity. In particular, this allows the M-branes of M-theory to be oxidised back to solutions in F-theory. The same twelve-dimensional theory also admits an inequivalent truncation, after dimensional reduction to $D=10$, that coincides in many respects with the bosonic sector of type IIB supergravity. Specifically, solutions of type IIB supergravity for which the bilinear quantity $\epsilon_{i j} d A_{2}^{(i)} \wedge d A_{2}^{(j)}$ vanishes will also be solutions of the dimensionally-reduced theory in $D=10$. One may hope that in configurations where the constraint vanishes, which can thus simultaneously be solutions of type IIB supergravity and the twelve-dimensional theory, the embedding again gives a valid oxidation of the lower-dimensional solutions to those of F-theory. The consistency of the truncation of fields in the ten dimensional theory requires the presence of a dilaton already in $D=12$, which, together with a linear combination $\varphi$ of the Kaluza-Klein dilatons $\phi_{1}$ and $\phi_{2}$, forms a complex scalar field $u$ that plays a rather unusual rôle. Specifically, the truncation allows $u$ to be set to zero, while $\bar{u}$ is required to be non-vanishing, although it is non-dynamical. It does, however, participate in the oxidation of solutions back to $D=12$.

The main defect of the embedding scheme that we have presented in this paper is that the theory in $D=10$ to which the compactified twelve-dimensional theory can be truncated is not quite the same as type IIB supergravity. However, its solution set has a considerable overlap with the solutions of the type IIB theory, including all of the BPS-saturated $p$-brane solitons in $D \leq 10$. One may hope that this lacuna can be overcome in a way that does not spoil this already successful embedding of the BPS solitons. This will be the case provided that the complete embedding differs from the one that we have presented here by terms that vanish when $\epsilon_{i j} d A_{2}^{(i)} \wedge d A_{2}^{(j)}$ is zero. Possible ideas for obtaining an exact embedding of the type IIB theory include introducing a Chern-Simons type modification of the form 
$G_{5}=d B_{4}+\kappa *\left(A_{3} \wedge d A_{3}\right)$ in $D=12$, which would lead to a modified 5-form field strength of the form $G_{5}=d B_{4}+\kappa *\left(\epsilon_{i j} A_{2}^{(i)} \wedge d A_{2}^{(j)}\right)$ in $D=10$. Another rather similar idea is to include a 7 -form field strength already in $D=12$ (again with a dilaton coupling with $\Delta=4$ ), which could take the form $G_{7}=d B_{6}+\kappa A_{3} \wedge d A_{4}$. In fact, neither of these possibilities seems to give the desired result in $D=10$, although they both show some promising features. In any case, provided that a complete description of the type IIB embedding can be found by some modification along these lines, the discussion of the oxidation of type IIB $p$-branes to $D=12$ in the previous section should continue to be valid. A further issue that we have not tackled in this paper is the inclusion of a fermionic sector in the twelve-dimensional theory. It may well be that this would single out a preferred signature for the additional 12 'th coordinate, which in our present discussion could be either spacelike or timelike.

Another unexplained aspect of the reduction procedures that we have described in this paper concerns the truncations of fields that we needed to perform in $D=11$ and $D=10$. Although we do not have a completely satisfactory explanation for why they should be performed, it is worth emphasising that there are rather tight constraints on what sets of fields can be consistently truncated from the dimensionally reduced theories. As we saw in $D=11$, the interaction terms in the Lagrangian imply that entire sets of fields must be truncated simultaneously, and so the number of possible truncations is severely limited.

A further observation that puts the truncations on a sounder basis is that there is in fact a symmetry principle that selects the fields that are set to zero in (11) and (21). Let us consider the eleven-dimensional case first. It is easy to see that after imposing the conditions (13) on the dilaton couplings in $D=12$, then substituting the definitions of $w$ and $\bar{w}$ into the full eleven-dimensional Lagrangian (8), we shall find that the fields listed in (11) will be precisely the ones whose exponential prefactors in (8) will include $\bar{w}$ dependence. It follows that the Lagrangian (8) has a global scaling symmetry in which we send $\bar{w} \rightarrow \bar{w}+$ const. together with appropriate non-trivial scalings of all the fields listed in the truncation (11). On the other hand the other fields that we are retaining in the Lagrangian (14) are invariant under this symmetry. Now it is always the case that if one truncates the fields in a Lagrangian to a subset comprising all the singlets under a symmetry group, then the truncation will be consistent [22]. This is because the singlet fields that are retained cannot act as sources for the non-singlets that are truncated. Similarly, in $D=10$ the set of fields that are truncated in (21) are precisely the subset that scale non-trivially under a global symmetry where $\bar{u} \rightarrow \bar{u}+$ const. in the full $D=10$ Lagrangian (10). The fact that there is a symmetry principle in both $D=11$ and $D=10$ that selects the truncated 
fields implies that one may hope that the consistency of these truncations will also persist beyond the level of the classical field theory.

We have described a twelve-dimensional theory that contains more bosonic degrees of freedom than are seen in its M-theory or type IIB theory dimensional reductions, thus necessitating the imposition of consistent truncations in $D=11$ or $D=10$. Of course one could take an alternative viewpoint, and impose precisely these field truncations already in $D=12$. Needless to say, they cannot be imposed in a twelve-dimensionally covariant manner, and so in this kind of a formulation the field theory in $D=12$ would have only eleven-dimensional or ten-dimensional covariance. It may therefore be merely a matter of convenience as to whether one prefers to work with a covariant twelve-dimensional theory with extra degress of freedom, or non-covariant theories with the correct degrees of freedom. After including the fermionic sector, one may similarly have such a choice of descriptions. In a twelve-dimensionally covariant form, the theory would, for example, reduce to a nonsupersymmetric theory in $D=10$ that admitted a consistent truncation to $N=2$ type IIB supergravity. Alternatively, by sacrificing twelve-dimensional covariance, one might be able to construct a supergravity theory that was already supersymmetric in $D=12$. However, since the truncation needed to obtain eleven-dimensional supergravity is inequivalent to the truncation needed to obtain type IIB supergravity, it would seem that the two would only be unified in $D=12$ by taking the covariant twelve-dimensional theory as the starting point.

\section{Acknowledgment}

We are grateful to K. Benakli and M.J. Duff for useful discussions. 


\section{References}

[1] C.M. Hull, String dynamics at strong coupling, Nucl. Phys. B468 (1996) 113.

[2] C. Vafa, Evidence for F-theory, Nucl. Phys. B469 (1996) 403.

[3] E. Witten, String theory dynamics in various dimensions, Nucl. Phys. B443 (1995) 184.

[4] E. Cremmer, B. Julia and J. Scherk, Supergravity theory in eleven dimensions, Phys. Lett. B76 (1978) 409.

[5] I.C.G. Campbell and P.C. West, $N=2 D=10$ non-chiral supergravity and its spontaneous compactification, Nucl. Phys. B243 (1984) 112.

[6] M. Huq and M.A. Namazie, Kaluza-Klein supergravity in ten dimensions, Class. Quant. Grav. 2 (1985) 293.

[7] E. Bergshoeff, H.J. Boonstra and T. Ortin, $S$ duality and dyonic p-brane solutions in type II string theory, Phys. Rev. D53 (1996) 7206.

[8] S. Ferrara, R. Minasian and A. Sagnotti, Low-energy analysis of $M$ and $F$ theories on Calabi-Yau threefolds, hep-th/9604097.

[9] A.A. Tseytlin, Type IIB instanton as a wave in twelve dimensions, hep-th/9612164.

[10] S. Kar, D-branes and twelve dimensions, hep-th/9701117.

[11] H. Lü, C.N. Pope, E. Sezgin and K.S. Stelle, Stainless super p-branes, Nucl. Phys. B456 (1995) 669 .

[12] H. Lü and C.N. Pope, p-brane solitons in maximal supergravities, Nucl. Phys. B465 (1996) 127.

[13] M.J. Duff and K.S. Stelle, Multi-membrane solutions of $D=11$ supergravity, Phys. Lett. B253 (1991) 113.

[14] R. Güven, Black p-brane solutions of $D=11$ supergravity theory, Phys. Lett. B276 (1992) 49.

[15] J.H. Schwarz, Covariant field equations of chiral $N=2 D=10$ supergravity, Nucl. Phys. B226 (1983) 269. 
[16] J.H. Schwarz, An $S L(2, Z)$ multiplet of type IIB superstrings, Phys. Lett. B360 (1995) 13.

[17] G.T. Horowitz and A. Strominger, Black strings and p-branes, Nucl. Phys. B360 (1991) 197.

[18] M.J. Duff and J.X. Lu, The self-dual type IIB superthreebrane, Phys. Lett. B273 (1991) 409.

[19] G.W. Gibbons, M.J. Green and M.J. Perry, Instantons and seven-branes in type IIB superstring theory, Phys. Lett. B370 (1990) 1.

[20] N. Khviengia, Z. Khviengia, H. Lü and C.N. Pope, Intersecting M-branes and bound states, Phys. Lett. B388 (1996) 21.

[21] H. Lü and C.N. Pope, p-brane taxonomy, hep-th/9702086.

[22] M.J. Duff and C.N. Pope, Consistent truncations in Kaluza-Klein theories, Nucl. Phys. B255 (1985) 355. 